\title{
Valid Entropy-Enthalpy Compensation: Its True Physical-Chemical Meaning
}

\author{
E.B. Starikov ${ }^{1,2, *}$ \\ ${ }^{1}$ Institute for Theoretical Solid-State Physics, University of Karlsruhe, D-76128 Karlsruhe, Germany \\ ${ }^{2}$ Department of Physical Chemistry, Chalmers University of Technology, SE-412 96 Gothenburg, Sweden
}

\begin{abstract}
The notion of entropy-enthalpy compensation (EEC) is well known and hotly debated up to now. Nevertheless, any valid EEC phenomenon ought to have in sooth a definite physical-chemical sense. The roots of the latter are discussed here in detail.
\end{abstract}

Keywords: Enthalpy, entropy, compensation, free energy, exergy, availability, thermodynamics.

\section{INTRODUCTION}

All the strolling around the EEC notion in the recent literature definitely shows that, although unskillful processing of equilibrium-thermodynamic and/or kinetic experimental data might well produce "phantom EEC findings", the true and physically-chemically valid EEC is not only just extant, but it ought to represent a useful interpretational tool as well (see [1-11] and the references therein).

In our most recent previous communication on the theme [9], together with our earlier work (cf. [4, 6-8, 10, 11] and the references therein), we could manage to demonstrate that describing the EEC in the general format ought to be in full accordance with the conventional thermodynamics.

Consequently, the purpose of the present communication is not only to provide a thorough analysis of the actual physical-chemical roots under the valid EEC findings, but also to open a door to a detailed critical re-evaluation of the thermodynamic notions.

\section{Physical-Chemical Sense of the EEC Parameters}

Mathematically, the general case of EEC can be expressed as a linear regression of enthalpy $H$ on entropy $S$, that is,

$H=T_{c} S+a$,

where $T_{c}$ stands for the so-called "compensation temperature" and the constant a has energy dimension.

*Address correspondence to this author at the Institute for Theoretical SolidState Physics, University of Karlsruhe, D-76128 Karlsruhe, Germany;

Tel: +49 72168037 19; E-mail: starikow@tfp.uni-karlsruhe.de
In our work [4] we have proven a theorem, which states, that "a valid, non-trivial EEC is a necessary and sufficient condition for the existence of a hidden thermodynamic cycle". Besides, Eq. 1 can also be very easily derived, if we start considering some arbitrary smooth thermodynamic cycle and decompose it into a number of infinitesimal Carnot cycles, as suggested in the book [12]. Then, for each infinitesimal cycle, we may write as follows, assuming, as usually, that the processes under consideration are slow enough and reversible:

$d S=\frac{\delta Q}{T} ; \quad d U=\delta Q-p d V$,

where the first equation is the Clausius definition of entropy, the second equation just expresses the $1^{\text {st }}$ law of thermodynamics, $Q$ stands for heat, $U-$ for internal energy, $p$ - for pressure, $V$ - for volume and $\delta Q$ means the inexact (path-dependent) differential, as opposed to the exact (path-independent) differential, $d$. And, after eliminating the inexact differential from Eq. 2, we get:

$d U=T d S-p d V$

which is nothing more than the conventional (Clausius-) Gibbs equation. Here, it is important to stress that we have already presented the following mathematical framework as it is in our most recent work [9]. And now it is instructive to try revealing the interconnection between the proper mathematical scheme and the actual physical-chemical sense of the parameters involved, in particular.

Integrating the latter, provided that we are dealing with the isobaric-isothermal situation, i. e., ( $p=$ const and $T=$ const) and bearing in mind that $H=U+p V$ by definition, would immediately lead to Eq. 1. Indeed, assuming the isobaric-isothermal situation, we ought to 
arrange physically-chemically that both $p$ and $T$ are in effect externally controllable parameters, so that, mathematically seen, the true integration variable coming to mind first of all would now be the system's volume - $V$. Hence, after the pertinent indefinite integration of Eq. 3, we arrive at the following antiderivatives' relationship:

$U(V, \ldots)-U_{\text {const }}=T S(V, \ldots)-T S_{\text {const }}-p V(\ldots)$,

$U(V, \ldots)+p V(\ldots)=T S(V, \ldots)-T S_{\text {const }}+U_{\text {const }}$,

where, by comparing Eq. 4b with Eq. 1, we get: $a \equiv U_{\text {const }}-T S_{\text {const }}$ and $T \equiv T_{c}$, so that the physicalchemical sense of the "constant a with the energy dimension" in Eq. 1 is then definitely nothing more than just the value of the system's Helmholtz free energy, $F_{c}$, at the "compensation temperature" (and the corresponding system's volume).

Therefore, in effect, we may recast Eq. 1 in the following - apparently unexpected - way:

$F_{c}=H-T_{c} S$.

Remarkably, the novelty of Eq. 5 is that it demonstrates an unconventional intrinsic interrelationship between the thermodynamic potentials of Gibbs and Helmholtz - and also that it is readily comparable with the standard expression for the availability or exergy function, $E_{e}$ (see, for example, [13-16] and the references therein for the detailed discussion of the latter notion):

$E_{e}=H-T_{c} S$,

where we adopt that $F_{c} \equiv E_{e}$. And hence, our system under study could be described by its internal energy, entropy, pressure, temperature and volume. In general, the system and its surrounding aren't initially in thermodynamic equilibrium with each other, but the process under study ought to reconcile this gap and thus bring the both into the equilibrium state. The latter action definitely requires some work to be done, and if we are to take into account both the First and the Second Laws of thermodynamics, we immediately come to the following conclusion:

The system's exergy is the maximum amount of work to be done by the system (or on the system), to allow the onset of the thermodynamic equilibrium between the system and the surrounding [15].

This ought to immediately deliver the exact physical-chemical meaning of both the "compensation temperature" ( $T_{c}-$ it is then just the temperature of the system's surrounding - to be reached in striving for the thermodynamic equilibrium between the former and the latter) and the "compensation energy constant" (this is the maximum energy cost to be paid for introducing the thermodynamic equilibrium between the system and its surrounding).

Specifically, it should be noted here, that the actual prerequisite of all the above consideration is that the system and its surrounding might only exchange some work and some heat, but not the matter (so, the system appears to be closed). Hence, one has to take this important point into account, when using the EEC findings as an interpretational tool. For, if the process in question would also take place in an open system, and therefore be connected with any kind of matter exchange, then one would definitely need to take both different pressure conditions and possible diffusive processes into account. And this might require more extended experimental approaches - to improve the resulting EEC picture with respect to the pertinent pressure and/or concentration stipulations.

Interestingly, the above seemingly logically clear standpoint is actually not something completely new, but has apparently gotten lost somehow in the 'physical chemistry developments shuffle' (or likewise) ... There was a relatively recent and a really interesting discussion on the closely related theme in the Journal of Chemical Education (see [17-19] and the references therein).

The notions of availability and exergy mentioned above were in effect introduced already long ago in the field of engineering thermodynamics - but these were (and, regrettably, still are) not taken seriously by physicists and chemists ... The reason for such a discrepancy is rooted in the way Rudolf Clausius had in his days interpreted (or, it is much more appropriate to put it as 'over-interpreted') the ingenious model by Nicolas Léonard Sadi Carnot. Clausius had immediately recognized that Carnot's model introduces and describes some exceedingly general phenomenon and Clausius readily dubbed it 'entropy', but, regrettably, he couldn't really manage to properly formulate the true general modalities of the latter, by producing his well-known maxima: "Die Entropie der Welt strebt einem Maximum zu" "The entropy of the world tends to a maximum"). The latter appears to contain only one proper statement, namely, "The entropy tends to a maximum". However, our Universe as a whole has definitely nothing to do with some 
particular, specific physical-chemical process, taking place in the Universe.

The so-called 'Carnot's cycle' is just a hugely idealized model of a realistic engine - and in effect nothing more than this. The main great result reached by Carnot is that even in such an idealized situation, when the engine' working ends up in its genuine initial state - and has moreover seemingly no outside agents that could interfere its working - but is nonetheless not working with $100 \%$-efficiency. This results from some intrinsic, internal reasons, which do interfere the proper working. And Rudolf Clausius has immediately and absolutely correctly recognized the source of such a state of affairs, as well as the profound generality of that source.

But now, a good question to pose would be: whether it is possible to define the entropy (and so on) without any application to the Carnot's cycle? The proper solution to the latter problem had been suggested by Max Planck and was successfully carried out by a German engineer Ernst Schmidt [20] (cf. the English version of Schmidt's work, as translated and published by a USA-American engineer, Joseph Kestin [21]). To sum up, the answer to the above poser is positive - yes, sure - the entropy might well be introduced without any application to the Carnot's cycle as well...

Regretfully, even the reference to Max Planck in Ernst Schmidt's work could not help attract the colleagues' attention to his seminal result ... To the best of my knowledge, virtually no published treatise on thermodynamics does even mention that somebody tried to re-evaluate the physical relevance of the ingenious model by Carnot ... Noteworthy, some attempts to critically re-evaluate the conclusions by Clausius had been undertaken long ago by an eminent British physicist, Hugh Longbourne Callendar [22], but the citation intensity of his seminal work is also tending to zero ... Instead, the overwhelming majority of thermodynamics handbooks discuss in detail „reversible" and „adiabatic processes" which have in effect nothing to do with actual, real happenings, but had been introduced by Carnot in his overwhelmingly idealized model of a strictly cyclic process free of any possible external hindrances ...

Remarkably, the above seemingly logically very clear viewpoint as to the significance of the Carnot's model wasn't - and still isn't - widely recognized by physicists and chemists, cf. for example the well- recognized 'Holy Bible' of theoretical physics by two prominent - and well-recognized all over the world Soviet physicists, Lev Davidovich Landau and Evgeny Mikhailovich Lifshitz [23]. In $\S \S 19$ and 20 of their, Statistical Mechanics' they present a logically perfect and transparent discussion of the 'maximum' and ,mininum' thermodynamic work. Still, their discussion is based upon the absolute validity of the Carnot's cycle. Such a standpoint is leading away from a clear definition of what the entropy in fact is - of what reversibility/irreversibility is, of what physical/chemical equilibria in open/closed systems are ...

Contrariwise, the latter group of notions is clearly defined and discussed in detail (together with introducing the notions of the 'maximum', 'minimum' thermodynamic work - and availability) in the handbooks for engineering thermodynamics (one of the most recent examples can be found in [24]).

A very good example of how to cope with misinterpretations in thermodynamics has been presented in the most recent book by Ken Sekimoto [25]. Especially mentionable in connection with the present communication ought to be the paragraph 2.3.4 of the latter book, where the author persuasively stresses and analyzes a possible interrelationship between the EEC and phase transitions. A careful survey of diverse modern EEC data fully supports such a possibility [8].

\section{The physical meaning of entropy}

All the above considerations ought to allow a successful analysis of the actual entropy notion.

Specifically, Eq. 4 is in accordance with the wellknown fact that the entropy ought to be always defined up to an additive constant - this is immediately following from the fact that the entropy is proportional to the logarithm of the probability (according to the Boltzmann-Planck ingenious guess), as E. Fermi had mathematically-rigorously shown in his book [26]. Along with this, E. Fermi distinctly stated there: "Off course, it should be clearly understood that this constitutes no proof of the Boltzmann equation, since we have not demonstrated that a functional relationship between entropy and probability exists, but have merely made it appear plausible."

On the other hand, we see that the "universal competition between energy and entropy" persuasively demonstrated in the book [12] clearly manifests itself 
as the EEC, at least in some particular isobaricisothermal cases. For our present EEC topic it is but of immense significance at least to try clarifying the interesting and important point: "Well, what is then the entropy"? The latter but still appears to be a hotly debatable issue [10] ...

Anyway, when using the energy availability, exergy standpoint to interpret valid EEC findings, it becomes clear that by measuring the entropy of a system before and after any real (mostly irreversible) process it is found that the final entropy is always greater than the initial one. This is just the genuine statement of the Second Law of thermodynamics. Furthermore, according to the First Law of thermodynamics, not the energy itself can be lost, but its availability for performing external work, the efficiency of its usage for the latter, is anyway decreasing in the course of real processes, as a result of the entropic degradation.

To sum up, the entropy increase should then be nothing more than the exergy loss [13-16]. And the latter statement could in principle serve as the genuine physical-chemical basis of the true, valid EEC phenomena. Then, the general physical-chemical meaning of the entropy notion could be formulated as follows: the notion of 'entropy' ought to stand for all the real phenomena, which somehow oppose the actual driving forces of the real processes. This "struggle and unity of opposites", or, in other words, a kind of "YinYang"-relationship, is just the actual sense of the "universal competition between energy and entropy" [12] mentioned above. Moreover, the true thermodynamic equilibrium of a real process ought to be the equilibrium between the pertinent driving force and its 'entropic opposition' ...

It is instructive to compare all the above considerations with the conventional standpoint more or less implicitly present throughout the physicalchemical literature and clearly formulated in the book by a British chemist, A. D. Buckingham [27]. In the Chapter 4.8 of his book (entitled "The Physical Nature of Entropy") he writes as follows:

"The thermodynamic definitions of dS and of the integration constant $S_{0}$ do not give any direct physical insight into the nature of the function of state $S$ called the entropy. To gain this insight, it is necessary to delve into statistical thermodynamics; Boltzmann successfully did this, and defined the thermodynamic probability $\Omega$ such that

$$
\ldots S=k \ln \Omega(4.33) \ldots
$$

... A thorough account of $\Omega$ requires statistical considerations, and here we shall merely point out that it is possible to equate $\Omega$ with the degeneracy of the state, that is, with the number of different microscopic states consistent with the bulk parameters $U$ and $V$ in an isolated system, or $T$ and $V$ in a system at constant volume in contact with a thermostat. Thus in an isolated system, $\Omega$ is the number of ways the energy $U$ can be distributed among the very large number of molecules comprising the system; entropy is therefore identified with the 'disorder' in the system, and an increase in entropy is associated with an increase in disorder. At $0^{\circ} \mathrm{K}$ the system is in its ground state (that is, the state, or states, of lowest energy), and if the ground state is singlet (that is, if there is only one lowest state) $\Omega=1$ and equation (4.33) gives $S_{0}=0$, in agreement with the third law."

Here we won't dwell on the pertinent details of the proper statistical considerations - they are contained in the book [28]. But it is anyway noteworthy that a) the above-cited, well-known Boltzmann-Planck formula was ingeniously guessed and never formally derived by its authors; b) building a mathematical parallel between $\Omega$ and the notion of probability isn't that trivial at all [28] ... A propos, as for the clear and realistic estimate of this really 'undeletable' parallelism between the entropy notion and 'disorder', see Frank L. Lambert's site [29].

Moreover, any appeal to "large numbers" isn't that insightful as well, for the term "large" is in effect fuzzy (operating with it necessarily entails the well-known "SORITES Paradox", "the Paradox of the Heap")... This is a serious conceptual problem of statistical mechanics, but we shall discuss it elsewhere.

To sum up, have we really learned anything about the physical nature of entropy from the above-cited text? Is then there some rational way out of this blind alley? ...

\section{The work of George Augustus Linhart}

Notably, already many years ago there was a successful - but completely unrecognized - attempt by Dr. George Augustus Linhart (1885 - 1951) to formally derive the Boltzmann-Planck expression using the above-mentioned purely logical approach to the probability notion - without any usage of the atomistic representation. This remarkable work could find its way to the attention of the scientific community only most recently (see $[30,31]$ and the references therein). In connection with $G$. A. Linhart's work, there is also one 
point of importance for our present EEC discussion. Specifically, Linhart had suggested a possible answer to the question: "What is entropy?" According to Linhart's ideas, entropy is nothing more than just 'all the possible hindrances to some real physical-chemical progress in question'. Anyway, in every real process, whatever it might be, there are some driving forces, which underlie and cause the 'progress', and there are inevitable and ubiquitous 'hindrances' as well, whereas the actual interplay between the latter both determines the actual outcome of the process under study.

Now, the interesting and important issue would be: How could we introduce the probability notion in this case? In view of the above-mentioned 'progresshindrance' dichotomy - or we might even say, 'progress-hindrance' dialectics - we definitely know that 'there ought to be at least some particular result of the process under study'. What we cannot faithfully foretell in many cases sounds this way: which exactly outcome might we get out of the process under study? Still, there certainly might be two possibilities (at the minimum!): we do get right what we expect - or just the contrary. To this end, speaking the probabilistic language, we may cast all this in such an assertion: The probability that we observe either the outcome $A$ or the outcome $B$ is equal to 1 , the probability that we observe neither $A$ nor $B$ is equal to 0 , whereas the probability that we observe exactly $A$ is equal to $0 \leq p \leq 1-$ and the probability that we observe exactly $B$ is equal to $1-p$. Hence, the task for the researcher is now to somehow find the formal mathematical expression for the $p$ and use it in solving further tasks ... This is, in effect, just what has been accomplished by Dr. G. A. Linhart. Moreover, he could really extend the meaning of the entropy notion starting from its conventional thermodynamic definition (what we know as the Clausius' formula) - and thus formally embody the well-recognized 'entropy's anthropomorphism' [10] $\cdots$

\section{CONCLUSIONS}

Bearing in mind all the above discussion, it is very important to stress that the conventional physicalchemical systems in question are in fact never isolated - they are always interacting with their respective surroundings - this important conceptual standpoint gets forgotten sometimes. Besides, the reversibility in physical-chemical processes is only approachable, but not reachable [32]. Hence, any realistic entropy increase - which is nothing more than just the loss of utilizable energy in the course of the pertinent process
- ought to occur in the system under study and its surrounding taken together. With this in mind, any valid entropy-enthalpy compensation finding describes the onset of thermodynamic equilibrium between the system and its surrounding. This is why, the conventional, so-called 'equilibrium', thermodynamics as well as the conventional, so-called 'equilibrium', statistical mechanics - ought to be carefully reconsidered.

\section{REFERENCES}

[1] Liu L, Guo QX. Isokinetic Relationship, Isoequilibrium Relationship, and Enthalpy-Entropy Compensatio. Chem Rev 2001; 101: 673. http://dx.doi.org/10.1021/cr990416z

[2] Cooper A, Johnson CM, Lakey JH, Nöllmann M. Biophys Chem 2001; 93: 215. http://dx.doi.org/10.1016/S0301-4622(01)00222-8

[3] Cornish-Bowden A. Enthalpy-entropy compensation: a phantom phenomenon. J Biosci 2002; 27: 121. http://dx.doi.org/10.1007/BF02703768

[4] Starikov EB, Nordén B. Enthalpy-Entropy Compensation: A Phantom or Something Useful? J Phys Chem B 2007; 111: 14431.

http://dx.doi.org/10.1021/jp075784i

[5] Lambert FL, Leff HS. The Correlation of Standard Entropy with Enthalpy Supplied from 0 to $298.15 \mathrm{~K}$. J Chem Educ 2009; 86: 94.

http://dx.doi.org/10.1021/ed086p94

[6] Starikov EB, Nordén B. Chem Phys Lett 2012; 538: 118. http://dx.doi.org/10.1016/j.cplett.2012.04.028

[7] Starikov EB, Nordén B. Entropy-enthalpy compensation may be a useful interpretation tool for complex systems like protein-DNA complexes: An appeal to experimentalists. Appl Phys Lett 2012; 100: 193701. http://dx.doi.org/10.1063/1.4714726

[8] Starikov EB, Nordén B. In: Current Microscopy Contributions to Advances in Science and Technology (A. Méndez-Vilas, Ed.) Microscopy Book Series $\mathrm{N}^{\circ}$ 5, vol. 2, Formatex Research Center, Badajoz, Spain 2012; p. 1492. http://www.formatex.info/microscopy5/book/1492-1503.pdf

[9] Starikov EB. J Appl Solution Chem Model 2012; 2: 126.

[10] Starikov EB. January 2013, Volume 144, Issue 1, pp 97-102

[11] 'Entropy is anthropomorphic': does this lead to interpretational devalorisation of entropy-enthalpy compensation? Monatshefte Chem 2013; 144 : 97. http://dx.doi.org/10.1007/s00706-012-0837-y

[12] Starikov EB. Chem Phys Lett 2013; 564: 88. http://dx.doi.org/10.1016/j.cplett.2013.02.016

[13] Müller I, Weiss W. Entropy and Energy: A Universal Competition 2005 Springer, Berlin \& Heidelberg, Germany.

[14] Bruges EA. Available Energy and the Second Law Analysis 1959 Butterworths Scientific Publications, London, Great Britain.

[15] Ahern JE. The Exergy Method of Energy Systems Analysis 1980 John Wiley \& Sons Inc., New York, Chichester, Brisbane, Toronto.

[16] Fricke J, Borst WL, Energie. Ein Lehrbuch der physikalischen Grundlagen 1981 R. Oldenbourg, München \& Wien.

[17] Sandler SI. Chemical, Biochemical and Engineering Thermodynamics 2006 John Wiley \& Sons Inc., New Delhi, India. 
[18] Lambert FL. Entropy Is Simple, Qualitatively. J Chem Educ 2002; 79: 1241.

http://dx.doi.org/10.1021/ed079p1241

[19] Jensen WB. Entropy and Constraint of Motion. J Chem Educ 2004; 81: 639 .

http://dx.doi.org/10.1021/ed081p639.2

[20] Lambert FL. Entropy and Constraint of Motion (author's response). J Chem Educ 2004; 81: 640. http://dx.doi.org/10.1021/ed081p640

[21] Schmidt E. Einführung in die thechnische Thermodynamik 1950 Springer-Verlag, Berlin, Göttingen, Heidelberg, Germany.

[22] Schmidt E. Thermodynamics. Principles and Applications to Engineering 1949 At the Carendon Press, Oxford, Great Britain.

[23] Callendar HL. The Caloric Theory of Heat and Carnot's Principle. Proc Phys Soc London 1910; 23: 153. http://dx.doi.org/10.1088/1478-7814/23/1/315

[24] Landau LD, Lifshitz EM, Pitaevkii LP. Course of Theoretical Physics. Vol. 5. Statistical Physics. Part. 1. 1980 Pergamon Press, Oxford, New York, Toronto, Sydney, Paris, Frankfurt.

[25] Elliott JR, Lira CT. Introductory Chemical Engineering Thermodynamics 2012 Pearson Education International, New York, Toronto, Montreal, London, Munich, Paris, Capetown, Sydney, Tokyo, Singapore, Mexico City.
[26] Sekimoto K. Stochastic Energetics 2010 Springer-Verlag, Berlin, Heidelberg, Dordrecht, London, New York. http://dx.doi.org/10.1007/978-3-642-05411-2

[27] Fermi E. Thermodynamics 1956 Dover Publications, New York, USA.

[28] Buckingham AD. The Laws and Applications of Thermodynamics 1964 Pergamon Press, Oxford, London, Edinburgh, Frankfurt, Paris - and - The Macmillan Company, New York, USA.

[29] Lavenda BH. Statistical Physics: A Probabilistic Approach 1991 John Wiley \& Sons, New York, Chichester, Brisbane, Toronto, Singapore.

[30] Dr. Frank L. Lambert's site about entropy: http://entropysite.oxy.edu/

[31] Starikov EB. Many Faces of Entropy or Bayesian Statistical Mechanics. Chem Phys Chem 2010; 11: 3387-94. http://dx.doi.org/10.1002/cphc.201000583

[32] Starikov EB. World J Cond Matt Phys 2012; 2: 101-16.

[33] Kline SJ. The Low-Down on Entropy and Interpretive Thermodynamics 1999 DCW Industries, Inc., California, USA.

Received on 09-11-2013

Accepted on 22-11-2013

Published on 28-11-2013

DOI: http://dx.doi.org/10.6000/1929-5030.2013.02.04.5

(C) 2013 E.B. Starikov; Licensee Lifescience Global.

This is an open access article licensed under the terms of the Creative Commons Attribution Non-Commercial License (http://creativecommons.org/licenses/by-nc/3.0/) which permits unrestricted, non-commercial use, distribution and reproduction in any medium, provided the work is properly cited. 